\title{
Potential Predictors for HPV Vaccination Completion Rates
}

Arushi Sachdev ${ }^{1,2}$, Kerman Sekhon ${ }^{3}$.

1 Department of Immunology, University of Toronto (St. George)

2 Department of Human Biology, University of Toronto (St. George)

3 Department of Biology, University of Toronto (Mississauga)

3- Pierre Elliott Trudeau High School (Markham, Ontario, Canada)

\section{Abstract}

Background: Human papillomavirus (HPV) is the most common sexually transmitted pathogen; its ease in transmission significantly contributes to its prevalence within the population. While most HPV infections are asymptomatic, a subset of infections cause genital warts, cervical cancer and various anogenital cancers. Accordingly, the vaccine Gardasil has been designed to prevent HPV infection and its associated sequelae. While Gardasil is effective against over $75 \%$ of cervical cancers, recent studies have demonstrated its limited adoption. In 2010, only $49 \%$ of females between the ages of 13-17 had received at least one dose. Moreover, Gardasil is a three-dose vaccine, and consequently, female patients that initiate the vaccination series often do not complete it in its entirety.

Methods: Data obtained from researchers at the Johns Hopkins Medical Institutions (JHMI) was used to determine which socioeconomic factors influence a female's likelihood of vaccination completion. The dataset consisted of female patients between the ages of 11-26 that had received at least one of the Gardasil vaccine doses from a JHMI clinic in Baltimore, USA, between the years 2006 and 2008. First, three logistic regression models were run with vaccination regimen completion, one shot completed and two shots completed as the dependent variables. Then, three LASSO logistic regression models were run to find relationships that were not influenced by model overfitting. The two regression methods were compared to determine if different results could be achieved.

Results: For the logistic regression, findings revealed that black females $(\mathrm{P}=0.006881)$, females between the ages of $18-26(\mathrm{P}=$ $0.000483)$, and females that visited urban clinics $(P=0.004582)$ are at an increased risk of incomplete vaccinations. In contrast, females that were treated by obstetrician-gynecologists $(\mathrm{P}=$ 0.006269 ) had increased compliance with the Gardasil vaccination regimen compared to women that visited other healthcare professionals. For the LASSO logistic regression, the model that penalized the most for overfitting showed that black females have a higher likelihood of only receiving one shot.

Conclusion: Due to the retrospective nature of the data, no causation can be established. However, these correlations shed light on what female populations should be studied further and potentially targeted to improve Gardasil vaccination completion rates. Moreover, the differences in vaccination completion rates can, in turn, aggravate the existing disparities in cervical cancer risk among females.

\section{Key Words}

Human Papillomavirus, Gardasil, Logistic Regression, LASSO Regression

\section{Introduction}

Human papillomavirus (HPV) is a virus that has over 100 strains, of which approximately 40 are sexually transmitted. ${ }^{1}$ While the majority of infections are asymptomatic or self-limiting, there is the risk of the infection progressing to cervical cancer, anogenital cancer, and/or genital warts. ${ }^{2}$ Due to such comorbidities, the rapid dissemination of the virus is concerning. Epidemiological studies have estimated that 6.2 million individuals between the ages of 14-44 become infected with HPV every year.1 Furthermore, by using these incidence rates, computational models have predicted that $80 \%$ of sexually active 50-year-old women are infected with genital HPV. ${ }^{3}$ Given HPV's prevalence, many women are at risk of developing infection-related morbidities, and as a result, it is no surprise that cervical cancer is ranked the second leading cause of death by cancer among women. ${ }^{4}$

In the interest of limiting HPV infection, in 2006, the vaccine Gardasil (Merck Labs) was approved and licensed by the United States Food and Drug Administration (FDA). The vaccine is quadrivalent, effective against HPV strains 6, 11, 16, and 18. To maximize effectiveness, the vaccination regimen requires three shots in total, with the second and third vaccinations occurring at the 
second and sixth month, respectively. ${ }^{5}$ Upon completion, Gardasil is protective against approximately $75-80 \%$ of all cervical cancers. ${ }^{6}$

Despite being the first vaccine that prevents cancer, Gardasil has not been widely received by the young female population. For instance, from a 2010 U.S. national survey, the Centers for Disease Control and Prevention (CDC) determined that only 49\% of females between the ages of 13-17 had received at least one dose. $^{7}$ Of those females, only $32 \%$ had received all three doses. ${ }^{7}$ The vaccination completion rates for Gardasil are low, and public health strategies must be implemented to address this problem.

Accordingly, the objective of this study was to analyze several socioeconomic variables that influence a female's likelihood of completing the Gardasil regimen. The statistical method selected needed to account for the fact that socioeconomic variables tend to be highly correlated. The reason being that when two or more variables are collinear, it is difficult to distinguish their respective predictive power on the response variable. ${ }^{8-10}$ When statistical models are vulnerable to multicollinearity, they run the risk of having unstable and/or inflated parameter and standard error estimates. ${ }^{9}$

Consequently, this study implemented a stepwise logistic regression in addition to a Least Absolute Shrinkage and Selection Operator (LASSO) logistic regression. The latter model penalizes independent variables that are highly correlated by reducing the parameter estimate towards an absolute value of zero. ${ }^{11}$ This study is interested in seeing if the two models yield different results regarding the likelihood of completing the Gardasil regimen. We predict that the LASSO logistic regression will have a more conservative output compared to the basic stepwise logistic regression, as it will detect the highly correlated socioeconomic variables. As a result, less independent variables will be considered significant.

\section{Materials and Methodology \\ Obtaining Data and Cleaning Data}

The dataset used in this study was obtained from Professor Seth Spielman's educational website. It is an abridged version of the raw data produced by the researchers at Johns Hopkins Medical Institutions (JHMI).12 The information in the dataset was acquired from young female patients (11-26 year olds) that received at least one of the Gardasil vaccine doses from a JHMI clinic in Baltimore, USA between the years 2006 and 2008.12 In total, there were 1,413 female patients. The dataset included the following variables: Age, Age Group (11-17 year olds or 18-26 year olds) Race (White, Black, Hispanic, Other), Number of Shots
Completed (1, 2 or 3), Completion of Gardasil Vaccine Regimen, Type of Insurance (Privately Paid, Hospital-Based Insurance, Military or Governmental Medical Assistance), Location of Clinic (Odenton, White Marsh, Johns Hopkins Outpatient Center, Bayview Medical Center), Clinic Location Type (Suburban or Urban), and finally, Type of Practice Female Patient Visited (Pediatric, Family Practice, OB-GYN).

First, using Microsoft Excel, the categorical variables in the dataset were converted to dummy variables to facilitate analysis and the formation of interaction terms. The variables OneShot, TwoShot and Completed were not included in interaction terms because they would each serve as dependent variables in their respective analyses. Interactions between the measured socioeconomic variables are possible, and accordingly, the relationships were further investigated by creating interaction terms. For instance, the race of a patient and their type of health insurance can have a synergistic effect. Similarly, the race of a patient and whether they visited a suburban or urban clinic may be correlated. Lastly, the patient's age group was included in a number of interaction terms. If interested in an exhaustive list of the variables analyzed in this study, please refer to the Appendix.

\section{Descriptive Statistics}

The R package ggplot2 was used to visualize the basic features of the dataset such as spread, variability and percentages. Since visualizing the data does not account for confounding variables, multivariable logistic regression was performed thereafter.

Backwards Stepwise Akaike Information Criterion Logistic Regression

A logistic regression was performed for statistical analysis because the dependent variables OneShot, TwoShot and Completed are all binary. Since the dataset had over 30 variables, there was a risk that the resulting logistic regression model would contain insignificant variables. Accordingly, to avoid overfitting and multicollinearity, the Backwards Stepwise Akaike Information Criterion (AIC) Selection method was used. The procedure involves first creating a model with all the predictor variables and calculating the respective AIC value. Then, in a sequential manner, one predictor variable is removed and the AIC value is recalculated. The logistic regression model with the lowest AIC value is used for analysis.

This process occurred three separate times, each with a different dependent variable -OneShot, TwoShot and Completed. For the 
logistic regression, variables with a p-value less than 0.05 were considered significant. If interested in the R output for this method, please refer to the Appendix.

\section{LASSO Logistic Regression}

As the dataset had thirty-four variables, the logistic regressions executed were susceptible to overloading predictor variables. Therefore, there was a risk of the three models demonstrating relationships that were not genuine due to overfitting. To circumvent this problem, a LASSO regression was performed to penalize the model whenever independent variables were highly correlated and did not contribute to the model. The magnitude of the penalty depends on the lambda value chosen, where lower values are more liberal and higher values are more conservative. Like before, a LASSO logistic regression was run for the three separate dependent variables. If interested in the $\mathrm{R}$ output for this method, please refer to the Appendix.

\section{Results}

\section{Descriptive Statistics}

The 1,413 female participants were grouped into two age groups - 11-17 year olds and 18-26 year olds. The division was almost equal, with $49.6 \%$ of the participants being categorized in the younger group and the remaining $50.4 \%$ being categorized in the older group. The median age for the 11-17 year olds group was 15 , and the median age for the 18-26 year olds group was approximately 21 . The wider spread of the older group was simply a reflection of the group's greater age range. Lastly, the unequal whiskers for the younger group show that their participants are skewed towards older ages. Refer to Figure 1 for the respective boxplot.

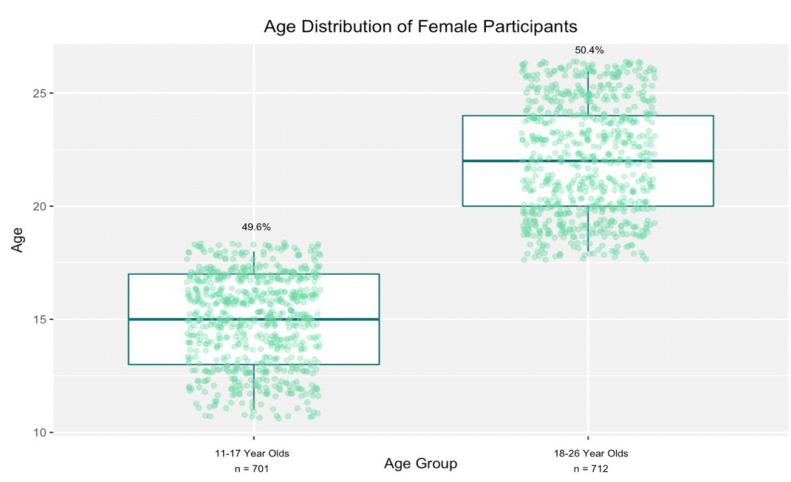

Figure 1: Age Distribution of Female Participants

The 1,413 female participants were not distributed equally in terms of race. The participants were predominantly White
(51.8\%), followed by Black (31.4\%) and then Hispanic (3.7\%). The remaining participants were categorized as "Other" (13.1\%). This racial distribution is observable in Figure 2.

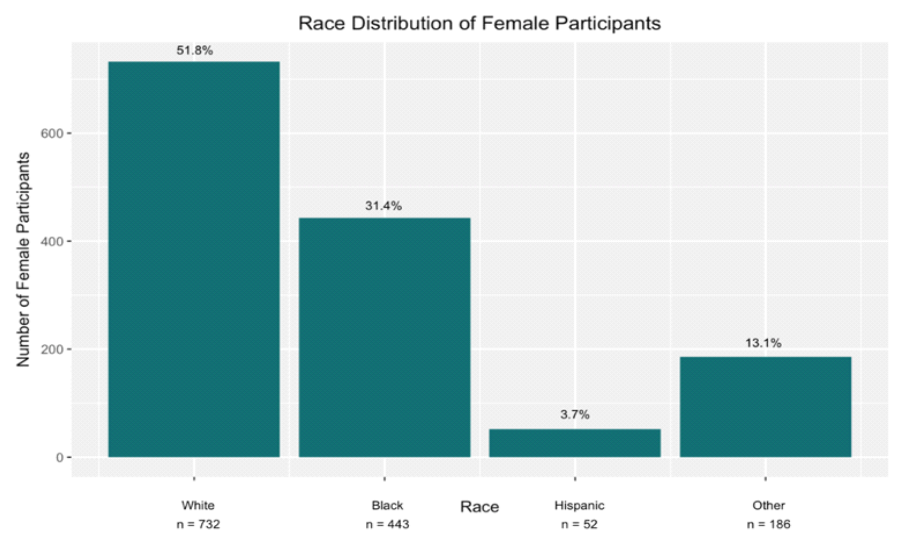

Figure 2: Race Distribution of Female Participants

As mentioned earlier, to complete the Gardasil regimen correctly, females must receive three shots within twelve months. The 1,413 female participants were categorized by how many Gardasil shots they received. To avoid redundancy, those that received three shots were not considered for the two-shot and one-shot categories, and similarly, those that received two shots were not considered for the one-shot category. Sixty-two percent of female participants ended their Gardasil regimen prematurely at the one-shot and two-shot stage. The remaining 38\% received three shots; however, this finding does not necessarily equate to the completing the vaccine regimen correctly. In fact, $13 \%$ of the females that received three shots received their third shot beyond the twelve-month period, thereby making the vaccination regimen less effective. These distributions are all observable in Figure 3.

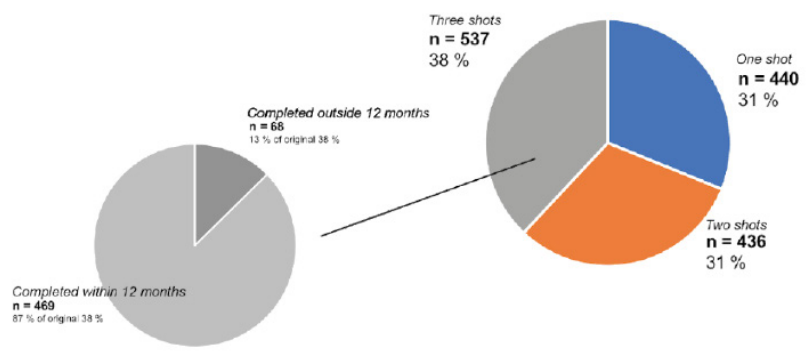

Figure 3: Not all females who receive three shots complete the Gardasil vaccination regimen correctly.

Backwards Stepwise Akaike Information Criterion (AIC) Logistic Regression

Three separate logistic regressions were performed to 
determine if there were potential predictor variables for: 1) the likelihood of completing the Gardasil regimen; 2) the likelihood of only receiving one shot, and; 3 ) the likelihood of only receiving two shots. Each logistic regression started with 34 variables, and after the backwards stepwise method, a number of variables were removed. The remaining predictor variables and their respective statistical significances are outlined in Table 1.

The multivariate analysis revealed several trends with regards to Gardasil vaccination completion. For example, the older the female participant (Age) is, the less likely they are to complete the regimen ( $\mathrm{P}=0.042795)$ and were more likely to stop at the two shots stage $(\mathrm{P}=0.00649)$. This observation was further supported by the older age group (X18.26) being strongly correlated to only receiving one shot of the vaccine $(\mathrm{P}=0.000483)$.

Another trend was that Black female participants were less likely to complete the Gardasil regimen ( $\mathrm{P}=0.006881)$, especially if they visited urban clinics in Baltimore (BlackUrban, $\mathrm{P}=$ 0.002511). However, there are two subsets of the Black population that are more likely to complete the Gardasil regimen: older Black females (X18.26Black, $P=0.034092)$ and Black females that receive governmental medical assistance (BlackMedAssist, $\mathrm{P}=0.025268$ ). Interestingly, White females under governmental medical assistance (WhiteMedAssist) were also more likely to complete the Gardasil regimen $(\mathrm{P}=0.022921)$. However, in general, females with this aid are less likely to complete the Gardasil regimen (MedicalAssistance, $\mathrm{P}=0.000581$ ). This suggests that insurance affects females differently. Another manifestation of this is that Black female participants that privately pay for the Gardasil vaccine are more likely to end the regimen at the two-shot stage (Black Private, $\mathrm{P}=0.02087$ ).

Furthermore, regardless of race, female participants were less likely to complete their vaccination regimen if they visited an urban clinic (Urban, $\mathrm{P}=0.004582$ ). For instance, Black female participants were less likely to reach completion (BlackUrban, $\mathrm{P}=0.002511$ ), Hispanic female participants were more likely to end at the second shot stage (HispanicUrban, $\mathrm{P}=0.01170$ ), and White female participants were less likely to complete the regimen (WhiteUrban, $\mathrm{P}=0.012499$ ).

Lastly, the type of physician practice visited by the patient for the Gardasil vaccine had an impact on vaccination completion. Obstetrician-gynecologists had higher retention with their patients (OB.GYN, P = 0.006269). Therefore, the female participants were less likely to end at the second shot stage $(P=0.00052)$. A physician's influence was also observed in older patients that visit pediatric doctors (X18.26Pediatric). They tended to only receive one vaccination dose $(\mathrm{P}=0.049453)$.

Table 1: Influence of Predictor Variables on Gardasil Regimen Progress

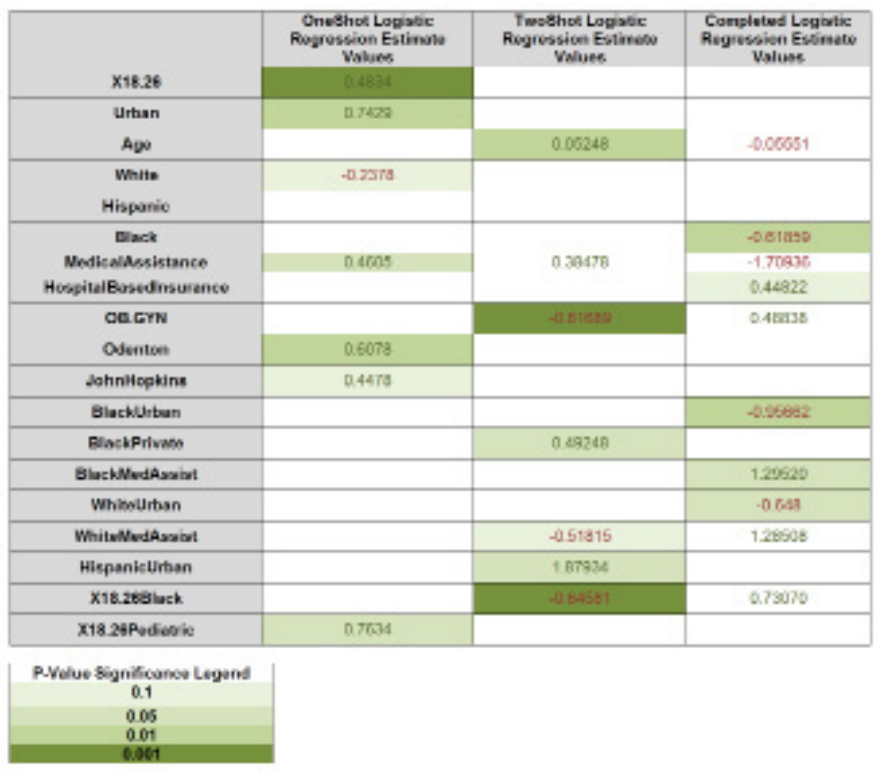

\section{LASSO Logistic Regression}

Three separate LASSO logistic regressions were run with completion of the Gardasil regimen as the dependent variable. The LASSO logistic regression with the minimum lambda value (0.007850481) penalized the model the least for additional variables, and consequently, a number of variables were deemed significant. However, when the LASSO logistic regression was run with the maximum lambda value $(0.06670973)$, no variables were deemed significant. As a result, the LASSO logistic regression with the average lambda value $(0.03728011)$ was run. It demonstrated that Black females who visited urban clinics in Baltimore and females with governmental medical assistance were less likely to complete the Gardasil regimen. In contrast, females that visited the White Marsh clinic were more likely to complete their Gardasil regimen. The $\mathrm{R}$ output does not provide $\mathrm{p}$-values, therefore, further analysis is required to determine how significant these results are. These relationships are observable in Table 2.

Three separate LASSO logistic regressions were run with receiving only one shot of the Gardasil regimen as the dependent variable. The LASSO logistic regression with the minimum lambda value $(0.002292268)$ penalized the model the least for additional variables, and consequently, a number of variables were deemed significant. When the LASSO logistic regression 
was run under the most conservative conditions (maximum lambda $=0.05420043)$, only the X18.26Black variable remained. This suggests that the relationship between older Black females and their increased likelihood of only receiving one shot may be important. As mentioned earlier, no p-value is linked to this predictor variable. As a result, it is difficult to determine how important the X18.26Black interaction term is.

Table 2: LASSO Regression with Completed as Dependent Variable

\begin{tabular}{|c|c|c|}
\hline Age & -0.040917829 & \\
\hline $\mathrm{X} 18.26$ & -0.166649056 & \\
\hline Urban & -0.078616478 & \\
\hline Black & -0.307881820 & -0.1363584 \\
\hline \multicolumn{3}{|l|}{ White } \\
\hline \multicolumn{3}{|l|}{ Hispanic } \\
\hline MedicalAssistance & -0.487052708 & -0.2080779 \\
\hline \multicolumn{3}{|l|}{ Privatelnsurance } \\
\hline HospitalBasedinsurance & 0.307686767 & \\
\hline \multicolumn{3}{|l|}{ Odenton } \\
\hline WhiteMarsh & 0.327652806 & 0.1629270 \\
\hline JohnHopkins & -0.020537604 & \\
\hline \multicolumn{3}{|l|}{ Pediatric } \\
\hline OB.GYN & 0.237699919 & \\
\hline BlackUrban & -0.379188154 & -0.1759369 \\
\hline \multicolumn{3}{|l|}{ BlackMedAssist } \\
\hline \multicolumn{3}{|l|}{ BlackPrivate } \\
\hline \multicolumn{3}{|l|}{ BlackHospital } \\
\hline \multicolumn{3}{|l|}{ WhiteUrban } \\
\hline \multicolumn{3}{|l|}{ WhiteMedAssist } \\
\hline WhitePrivate & 0.003634982 & \\
\hline \multicolumn{3}{|l|}{ WhiteHospital } \\
\hline \multicolumn{3}{|l|}{ HispanicUrban } \\
\hline HispanicMedAssist & -0.162072246 & \\
\hline \multicolumn{3}{|l|}{ HispanicPrivate } \\
\hline HispanicHospital & 1.669762580 & \\
\hline X18.26Pediatric & -0.372290587 & \\
\hline X18.260B.GYN & 0.074103728 & \\
\hline \multicolumn{3}{|l|}{ X18.26Black } \\
\hline \multicolumn{3}{|l|}{ X18.26White } \\
\hline $\mathrm{X} 18.26 \mathrm{Hispanic}$ & & \\
\hline
\end{tabular}

A LASSO logistic regression with the average lambda value $(0.02824635)$ was also run and shared some of the relationships observed the LASSO logistic regression run with the Completed dependent variable. For instance, it was previously observed that female participants who visited the White Marsh clinic were more likely to complete the Gardasil regimen. Here, it is shown that female participants that visited the White Marsh clinic were less likely to only receive one shot. Another example of shared observations was that female participants who receive governmental medical assistance were less likely to complete the Gardasil regimen and were more likely to only receive one shot. Once again, the $\mathrm{R}$ output does not provide $\mathrm{p}$-values, therefore, further analysis is required to determine how significant these results are. Other relationships are observable in Table 3.

Table 3: LASSO Regression with OneShot as Dependent Variable

\begin{tabular}{|c|c|c|c|}
\hline & lambda $=0.002292268$ & lambda $=0.02824635$ & lambda $=0.05420043$ \\
\hline Age & 0.003881171 & & \\
\hline$x 18.26$ & 0.392500147 & 0.10914557 & \\
\hline \multicolumn{4}{|l|}{ Urban } \\
\hline Black & 0.030204286 & & \\
\hline White & -0.196118467 & & \\
\hline \multicolumn{4}{|l|}{ Hispanic } \\
\hline MedicalAssistance & 0.454718114 & 0.16008246 & \\
\hline Privatelnsurance & 0.087294153 & & \\
\hline HospitalBasedinsurance & -0.175653242 & & \\
\hline \multicolumn{4}{|l|}{ Odenton } \\
\hline WhiteMlarsh & -0.540197352 & -0.14550846 & \\
\hline JohnHopkins & 0.382516787 & 0.17192759 & \\
\hline Pediatric & -0.062279159 & & \\
\hline \multicolumn{4}{|l|}{ OB.GN } \\
\hline BlackUrban & 0.157963744 & 0.08966726 & \\
\hline \multicolumn{4}{|l|}{ BlackMledAssist } \\
\hline \multicolumn{4}{|l|}{ BlackPrivate } \\
\hline \multicolumn{4}{|l|}{ BlackHospital } \\
\hline WhiteUrban & 0.127007901 & & \\
\hline WhiteMledAssist & 0.139419590 & & \\
\hline \multicolumn{4}{|l|}{ WhitePrivate } \\
\hline WhiteHospital & 0.277320189 & & \\
\hline Hispanicurban & -1.919416331 & & \\
\hline HispanictlledAssist & 1.332114480 & & \\
\hline HispanicPrivate & -0.644943294 & & \\
\hline HispanicHospital & -2.196236015 & & \\
\hline X18.26Pediatric & 0.693807779 & 0.20349800 & \\
\hline X18.260B.GYN & -0.061578547 & & \\
\hline X18.26Black & 0.114343715 & 0.18557947 & $3.829585 e-08$ \\
\hline \multicolumn{4}{|l|}{ X18.26White } \\
\hline X18.26Hispanic & 1.270748705 & & \\
\hline
\end{tabular}

Three separate LASSO regressions were run with receiving only two shots of the Gardasil regimen as the dependent variable. The LASSO logistic regression with the minimum lambda value (0.001047045) penalized the model the least for additional variables, and consequently, a number of variables were deemed significant. However, when the LASSO logistic regression was run with the maximum lambda value (0.03591857), no variables were deemed significant. As a result, the LASSO logistic regression with the average lambda value (0.01848281) was run. Interestingly, the relationships observed were not previously observed with the Completed and OneShot LASSO logistic regressions. Female participants that had hospital-based insurance and female participants that were treated by obstetrician-gynecologists were less likely to only receive two shots of the Gardasil regimen. In contrast, Hispanic females that visited urban clinics in Baltimore were more likely to only receive two shots of the Gardasil regimen. As mentioned earlier, due to the lack of p-values, further analysis is required to determine the significance of these predictor values. These relationships are outlined in Table 4.

\section{Discussion}

This study's analysis revealed that approximately a third of the female participants completed the Gardasil vaccination 
regimen within the recommended twelve months. The logistic regression and LASSO logistic regression helped determine potential predictor variables that could influence a female's likelihood in vaccination completion. We purposely used and compared two separate regression methods in this study in order to identify weaknesses with each approach and more confidently assess predictor variables. For example, the model for the logistic regression was determined by a backwards stepwise AIC method.

\begin{tabular}{|c|c|c|}
\hline & lambda $=0.001047045$ & lambda $=0.01848281$ \\
\hline Age & 0.05465661 & \\
\hline $\mathrm{X} 18.26$ & 0.04524944 & \\
\hline \multicolumn{3}{|l|}{ Urban } \\
\hline Black & 0.20918802 & \\
\hline \multicolumn{3}{|l|}{ White } \\
\hline Hispanic & -0.86340396 & \\
\hline MedicalAssistance & 0.18542950 & \\
\hline Privateinsurance & -0.04653899 & \\
\hline HospitalBasedinsurance & -1.37802579 & -0.01605313 \\
\hline Odenton & -0.27345241 & \\
\hline Whitellarsh & 0.01565902 & \\
\hline JohnHopkins & -0.26991062 & \\
\hline Pediatric & -0.22800962 & \\
\hline OB.GYN & -0.54363684 & -0.09776230 \\
\hline \multicolumn{3}{|l|}{ BlackUrban } \\
\hline \multicolumn{3}{|l|}{ BlackMedAssist } \\
\hline BlackPrivate & 0.29362642 & \\
\hline BlackHospital & 1.48058502 & \\
\hline WhiteUrban & 0.05878695 & \\
\hline WhitelMedAssist & -0.34801687 & \\
\hline \multicolumn{3}{|l|}{ WhitePrivate } \\
\hline WhiteHospital & 0.87527484 & \\
\hline HispanieUrban & 1.81947849 & 0.49185211 \\
\hline \multicolumn{3}{|l|}{ HispaniclMedAssist } \\
\hline \multicolumn{3}{|l|}{ HispanicPrivate } \\
\hline HispanicHospital & -1.82489381 & \\
\hline X18.26Pediatric & -0.29290566 & \\
\hline \multicolumn{3}{|l|}{ X18.260B.GYN } \\
\hline X18.26Black & -0.61563100 & \\
\hline X18.26White & -0.05450602 & \\
\hline X18.26Hispanic & & \\
\hline
\end{tabular}

Table 4: LASSO Regression with TwoShot as Dependent Variable

A different model could have been achieved if done forwards or if another criteria besides AIC was used. As for the LASSO logistic regression, it is difficult to determine how conservative the lambda should be. Furthermore, the LASSO logistic regression is not accompanied by p-values, which makes it difficult to determine how significant a predictor variable is for the dependent variable being analyzed. Accordingly, these results should encourage future studies to investigate the causality underlying these trends. In the subsequent paragraphs, the suggested relationships from our data analysis will be analyzed.

The data analysis suggests that the older the female patient, the more likely they will not complete the vaccination regimen. This may be because majority of young females have their parents or guardians book their medical appointments, and therefore, do not hold as much personal and financial responsibility in terms of their health. An older female has personal and financial obligations that may impede them from completing the vaccination regimen.

Another trend was that people who visited urban clinics were less likely to complete their vaccination regimen. To determine the underlying reasons for this trend, future studies should examine the ease of access and clinic wait times of both urban clinics and suburban clinics. In terms of physician practice, female participants that visited obstetrician-gynecologists tended to have higher compliance to the Gardasil regimen compared to those that visited family doctors or pediatricians. Future studies should determine if doctors of different specialities have different vaccination strategies or vary in how strongly they recommend getting vaccinated against HPV.

In terms of race, it was noted that, in general, Black female participants are at risk of an incomplete vaccination regimen. To interpret this relationship better, more socioeconomic variables should be included in the dataset such as household income, educational status or distance from clinic. That being said, the stepwise logistic regression model revealed two Black subpopulations that were more likely to complete their vaccination regimen: females between the ages of 18-26 and females that received government medical assistance. Yet, this phenomenon was not shared with the more conservative LASSO logistic regression. The model deemed that Black females between the ages of 18-26 are more likely to only complete one out of the three vaccinations. In fact, this was the only observation that was supported under the most penalized model after accounting for overfitting and multicollinearity. For future studies, adjustments to the dataset are recommended. As mentioned earlier, more socioeconomic variables would be beneficial. In addition, since each patient's personal identifiers have not been included in the dataset, it is difficult to determine if there are any duplicate entries. As a result, each patient should have a unique number associated with their entry for cross-referencing purposes. The reason why this would be beneficial is because there could be cross-over cases between the four clinics in Baltimore, USA. For example, the female patient may have visited the Bayview Medical Center for her first two shots and then Odenton for her last shot in the 
vaccination regimen. Is this patient inputted in the dataset twice? Or is the patient's information combined? If so, which variables would be used in these cross-over cases (e.g. suburban vs. urban, OB-GYN vs. pediatric vs. family)? Another source of error is that the data accumulated by JHMI assumes that the female patient revisits the same clinic for each subsequent Gardasil vaccination dose. For example, if a female visited the Odenton clinic for her first shot and then visited an out-of-city clinic for her subsequent two shots, the dataset would have reported her as an incomplete vaccination regimen.

\section{Conclusion}

While the merits of Gardasil are evident, the opportunity to limit HPV infection and cervical cancer is limited on two fronts: first, the low vaccination rates and second, the low rates of completion for females that do commence the Gardasil vaccination regimen. Through the use of data science, this study revealed certain female subpopulations that are more and less likely to complete the vaccination series, and in turn, these results can be used to direct more robust analyses. We are hopeful that future research will identify the vaccination behaviours within distinct female cohorts, and what effective strategies should be implemented to target these cohorts to increase overall vaccination completion rates. research.

Thank you to Aniruddh Sachdev for sharing his expertise in data science by being an advisor during our research.

Thank you to Emily Estes, Jayneelkumar Limbachia, Rahul Arora, Priscilla Ng, and Harmehr Sekhon for providing feedback and suggestions during the manuscript writing process.

\section{Acknowledgements}

Thank you to STEM Fellowship and IBM Big Data University for offering more than adequate guidance in completing this

\section{References}

1. Weinstock, H., Berman, S. \& Cates, W. Sexually Transmitted Diseases among American Youth: Incidence and Prevalence Estimates, 2000. Perspect. Sex. Reprod. Health 36, 6-10 (2004).

2. Markowitz, L. E. et al. Quadrivalent human papillomavirus vaccine. MMWR Morb Mortal Wkly Rep 56, 1-24 (2007).

3. Myers, E. R., McCrory, D. C., Nanda, K., Bastian, L. \& Matchar, D. B. Mathematical model for the natural history of human papillomavirus infection and cervical carcinogenesis. Am. J. Epidemiol. 151, 1158-1171 (2000).

4. Parkin, D. M., Bray, F., Ferlay, J. \& Pisani, P. Estimating the world cancer burden: Globocan 2000. Int. J. cancer 94, $153-156$ (2001).

5. Jackson, J., Braccio, N., Brevig, H. \& Sengupta, D. FDA Advisory Committee Trends Since FDASIA. 16 (2015). Available at: https://avalere-health-production.s3.amazonaws.com/uploads/pdfs/1448383851_112315_Avalere_AdCommFocusReport.pdf.

6. Petry, K. U. HPV and cervical cancer. Scand. J. Clin. Lab. Invest. 74, 59-62 (2014).

7. (CDC, C. for D. C. and P. National and state vaccination coverage among adolescents aged 13 through 17 years--United States, 2010. MMWR. Morb. Mortal. Wkly. Rep. 60, 1117 (2011).

8. Alin, A. Multicollinearity. WIREs Computational Statistics, 2. (2010).

9. Dormann, C. F. et al. Collinearity: a review of methods to deal with it and a simulation study evaluating their performance. Ecography (Cop.). 36, 27-46 (2013).

10. Meloun, M., Militký, J., Hill, M. \& Brereton, R. G. Crucial problems in regression modelling and their solutions. Analyst 127, 433-450 (2002).

11. Hastie, T., Tibshirani, R. \& Friedman, J. in The elements of statistical learning 485-585 (Springer, 2009).

12. Spielman, S. Logistic Regression Lab. (2017). Available at: http://www.sethspielman.org/courses/geog5023/labs15/Lab_2015_Logistic_Regression.html. (Accessed: 24th May 2017) 


\section{Appendix}

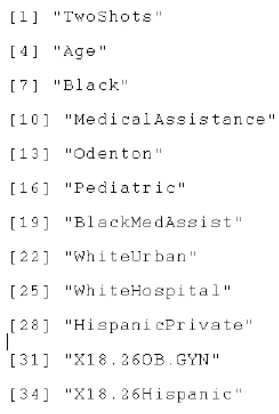

Variable

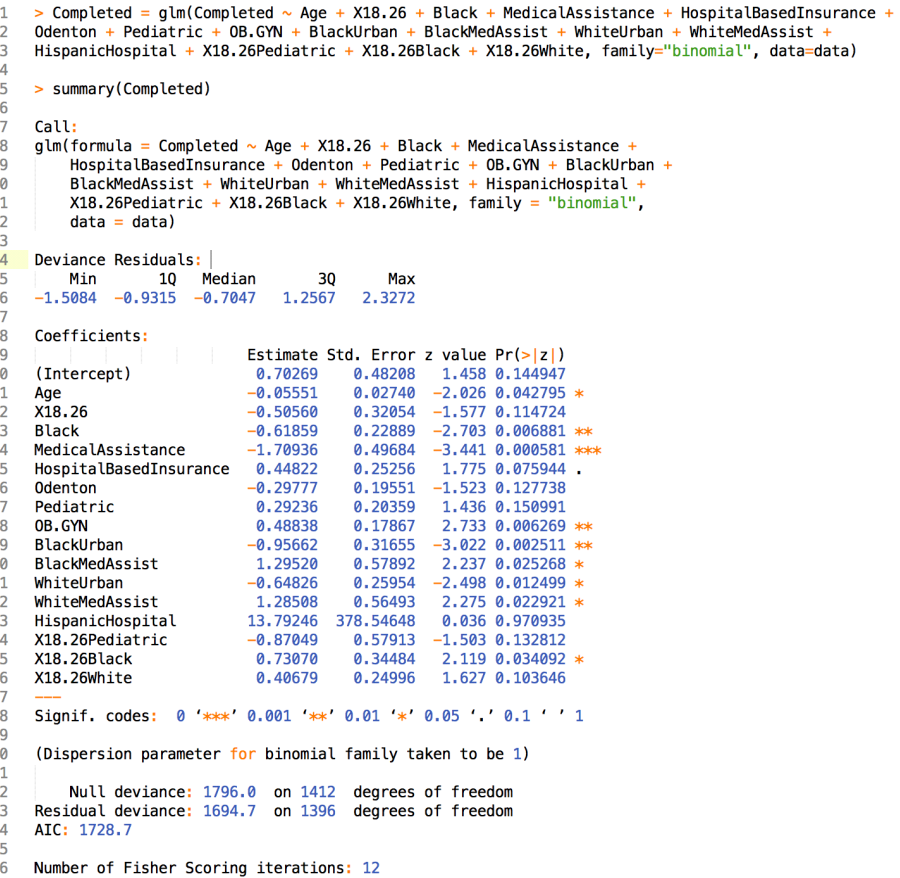

Number of Fisher Scoring iterations: 12 helped determine what variables should be included in the logistic regression model.

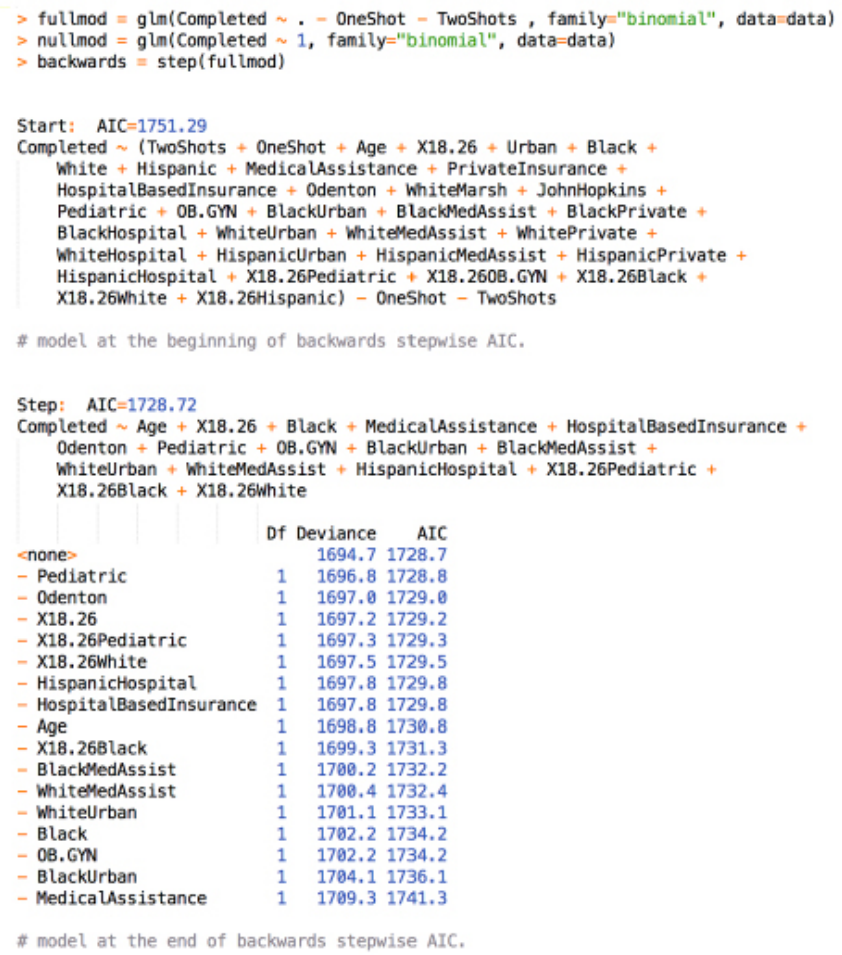

4. R OUTPUT: Logistic Regression with OneShot as Dependent Variable

6. R OUTPUT: LASSO Regression with Completed as Dependent

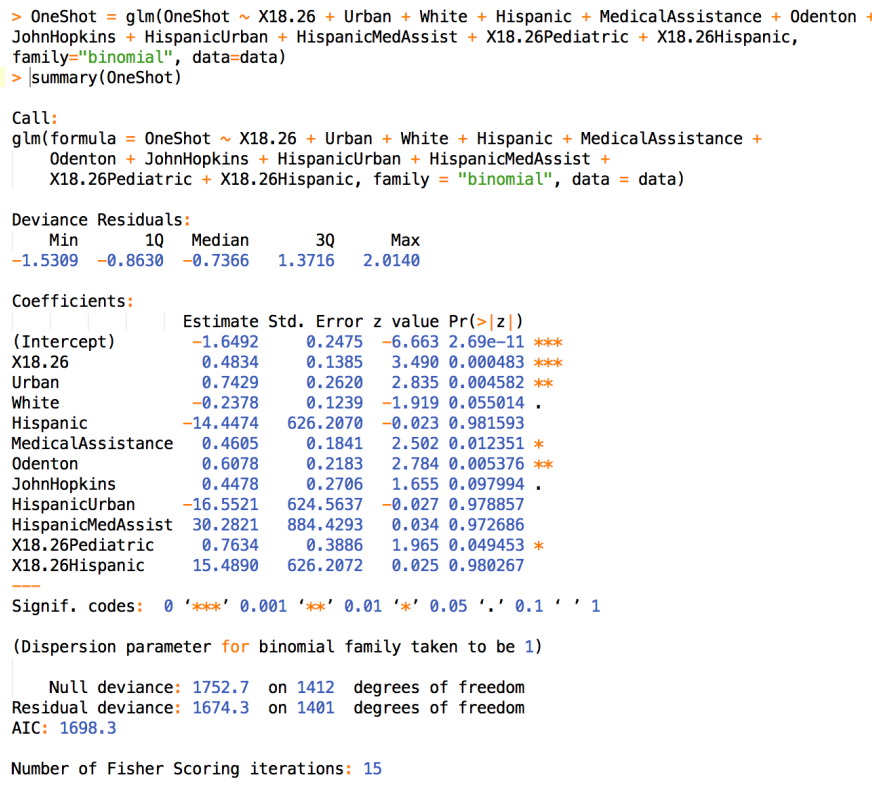

Variable

The following R output is for when lamba is at its minimum. 
